\title{
EXPERIENCIA DE LOS ESTUDIANTES QUE INGRESAN POR CUPOS DE EQUIDAD A LA ESCUELA DE OBSTETRICIA DE LA UNIVERSIDAD DE CHILE.
}

\section{EXPERIENCE OF STUDENTS WHO ENTER THEIR EQUITY AT SCHOOL OF OBSTETRICS OF THE UNIVERSITY OF CHILE.}

\author{
Andrea Velásquez-Muñoz ${ }^{1}$.avelasquez74@gmail.com \\ Francisco Bustamante-Toncio².franciscobt@gmail.com \\ Maribel Mella-Guzmán ${ }^{3}$.m.a.mellaguzman@gmail.com \\ Claudia Cornejo-Aravena ${ }^{4}$, claudiacornejoaravena@gmail.com \\ Universidad de Chile, Chile
}

\author{
Volumen 8, Número 2 \\ 30 de noviembre de 2017
}

pp. $200-219$

Recibido: 08 de julio de 2017

Aprobado: 30 de octubre de 2017

\footnotetext{
${ }^{1}$ Académico. Departamento de Promoción de la Salud de la Mujer y el Recién Nacido. Facultad de Medicina. Universidad de Chile. Correo electrónico: avelasquez74@gmail.com

${ }^{2}$ Académico. Departamento de Promoción de la Salud de la Mujer y el Recién Nacido. Facultad de Medicina. Universidad de Chile. Correo electrónico: franciscobt@gmail.com

${ }^{3}$ Académico. Departamento de Promoción de la Salud de la Mujer y el Recién Nacido. Facultad de Medicina. Universidad de Chile. Correo electrónico: m.a.mellaguzman@gmail.com

${ }^{4}$ Académico. Departamento de Promoción de la Salud de la Mujer y el Recién Nacido. Facultad de Medicina. Universidad de Chile. Correo electrónico: claudiacornejoaravena@gmail.com
}

Evaluación de la implementación de los cupos de equidad de la Escuela de Obstetricia de la Universidad de Chile.

Andrea Velásquez-Muñoz, Francisco Bustamante-Toncio

Maribel Mella-Guzmán, Claudia Cornejo-Aravena

DOI : http://dx.doi.org/10.22458/caes.v8i2.1839

Artículo protegido por licencia CreativeCommons 


\section{Resumen}

En la Universidad de Chile, desde el 2013 se ha implementado un nuevo sistema de ingreso para estudiantes de excelencia en la trayectoria académica que provienen de establecimientos públicos de alta vulnerabilidad. Objetivo: Conocer la experiencia de los estudiantes que ingresan por cupos de equidad a la Escuela de Obstetricia entre los años 2013 a 2016. Métodos: Estudio de casos. Se aplicó un cuestionario a los estudiantes y se realizaron entrevistas a los docentes. Resultados: La deserción fue del $41 \%$, cuya principal causa fue vocacional. El $90 \%$ de los estudiantes que aún permanecen presentan reprobación. De acuerdo a los estudiantes y docentes, los principales obstaculizadores fueron la falta de técnicas de estudio y el déficit de conocimientos en ciencias básicas. Conclusiones: La experiencia para los estudiantes es gratificante, pues les asegura el ingreso a la Universidad. Sin embargo, es frustrante para ellos darse cuenta de todas las falencias teóricas que tienen, producto de las desigualdades que existen en la educación en Chile. Es necesario repensar las estrategias de apoyo de manera que sean percibidas como ayuda y no como sobrecarga académica.

Palabras Clave: Inclusión; educación superior; acceso; permanencia.

\section{Abstract.}

At the University of Chile, since 2013 a new entrance system has been implemented for students of excellence in the academic trajectory that come from public establishments of high vulnerability. Objective: To know the experience of the students that enter by equity quotas to the School of Obstetrics between the years 2013 to 2016. Methods: Case studies. A questionnaire was applied to the students and interviews were held with the teachers. Results: The dropout rate was $41 \%$, whose main cause was vocational. $90 \%$ of the students who still remain present reprobation. According to the students and teachers, the main obstacles were the lack of study techniques and the lack of knowledge in basic sciences. Conclusions: The experience for the students is gratifying, since it assures them the entrance to the University. However, it is frustrating for them to realize all the theoretical shortcomings they have, as a result of the inequalities that exist in education in Chile. It is necessary to rethink the support strategies so that they are perceived as help and not as academic overload.

Key words: Inclusion; higher education; access; permanence.

Evaluación de la implementación de los cupos de equidad de la Escuela de Obstetricia de la Universidad de Chile.

Andrea Velásquez-Muñoz, Francisco Bustamante-Toncio Maribel Mella-Guzmán, Claudia Cornejo-Aravena DOI : http://dx.doi.org/10.22458/caes.v8i2.1839 


\section{Introducción}

La equidad en la educación superior, que se funda en el derecho de cada persona a contar con una educación de calidad que le permita desarrollar plenamente su potencial, plantea desafíos al Estado y a las instituciones, entre los cuales, está el garantizar que todos los individuos puedan alcanzar resultados educativos que les permitan insertarse en el mundo laboral y en el sistema social en igualdad de condiciones y derechos (Espinoza, González y Latorre, 2009).

Los estudios nacionales e internacionales indican que, entre los países de América Latina, Chile es uno de los que presentan una mayor desigualdad en la distribución de ingresos (Cepal, 2010; OCDE, 2009; Solimano y Torche, 2008). Dicha desigualdad encuentra su correlato en un sistema educativo altamente segmentado y estratificado (Bellei y Huidobro, 2003; OCDE, 2004; OCDE 2009; Valenzuela, Bellei y De los Ríos, 2009).

En base a lo anterior, en Chile se ha ido consolidando una visión que muestra una progresiva apertura de la educación superior a segmentos de la población, que antiguamente se encontraban excluidos de este nivel educativo, ya sea por razones socioeconómicas, de género, etnia, raza, sociales, culturales, entre otros (Castillo y Cabezas 2010). A pesar de ello, las universidades selectivas siguen reproduciendo las inequidades del sistema escolar al determinar la admisión, principalmente, en función del desempeño en la Prueba de Selección Universitaria (PSU), cuyos resultados se correlacionan con el nivel socioeconómico de los y las estudiantes (Contreras, Corbalán y Redondo, 2007).

La Universidad de Chile, en tanto universidad del Estado, en el contexto del diálogo por el nuevo trato para las universidades estatales, plantea "proveer opciones de educación superior 
para todos, haciéndose corresponsable en la tarea de superar la desigualdad en la educación en todos sus niveles, y crear las condiciones para que esa meta se mantenga en forma permanente" (Pérez, 2009). Este mandato supone revisar en las diversas carreras que componen a la universidad, tanto los mecanismos que de hecho operan para seleccionar estudiantes, así como también el aporte en el desarrollo de equidad educativa en el sistema total.

En base a lo anterior, surgen debates en torno a alternativas orientadas a revertir la desigualdad en la educación superior, lo que se ha traducido en el diseño e implementación de programas de ingreso complementario o alternativo a la PSU. Entre ellos, se encuentra la creación del Sistema de Ingreso Prioritario de Equidad Educativa (SIPEE) de la Universidad de Chile (Williams, 2013).

El Sistema de Ingreso Prioritario de Equidad Educativa (SIPEE) de la Universidad de Chile no solo supone un compromiso con la ampliación del acceso, sino también con la permanencia y el egreso de todos los estudiantes meritorios que aspiran a ser parte de la universidad y que provienen de distintas realidades sociales (Pérez, 2011). Desde una concepción que entiende que hablar de calidad supone atención a la diversidad, esta iniciativa se acompaña de acuerdos fundamentales que se orientan a promover "un conjunto de mejoras e innovaciones a la docencia e intensificar los apoyos al desempeño académico y la calidad de vida estudiantil", entre otras profundas transformaciones internas (Sobrero, Lara, Méndez, Suazo, 2014: 161).

El Sistema de Ingreso Prioritario de Equidad Educativa (SIPEE) consiste en la generación de cupos especiales de ingreso a distintas carreras, reservados para estudiantes provenientes de liceos municipales, que pertenezcan a los tres primeros quintiles de ingreso y que cuenten con

Evaluación de la implementación de los cupos de equidad de la Escuela de Obstetricia de la Universidad de Chile.

Andrea Velásquez-Muñoz, Francisco Bustamante-Toncio

Maribel Mella-Guzmán, Claudia Cornejo-Aravena

DOI : http://dx.doi.org/10.22458/caes.v8i2.1839 
el puntaje mínimo para postular ( 600 puntos en la PSU). Entre todos los postulantes, se realiza una selección, donde se prioriza el Índice de vulnerabilidad de la escuela de origen, luego el quintil de ingreso de la familia del estudiante y en tercer lugar, si es perteneciente al $10 \%$ de los mejores promedios de su liceo.

La carrera de Psicología de la Universidad de Chile es pionera en la Universidad, en implementar a partir del año 2010,la creación de un tipo de ingreso especial que se le ha denominado "cupo de equidad", cuyas características apuntan a favorecer el ingreso de estudiantes de los tres primeros quintiles y de liceos municipales. Su implementación ha demostrado que logra el efecto buscado, que es intervenir en la tendencia que se apreciaba en la carrera desde el año 2003, una progresiva disminución de jóvenes del sector municipal. De esta forma, para el año 2010, se equiparó el número de estudiantes provenientes de establecimientos de los tres tipos de dependencia administrativa existentes en el sistema escolar (Castro, Antivilo, Aranda, Castro, de Torres, Lizama, Williams, 2012). A los dos años de implementados los cupos de equidad, en la carrera de Psicología, se puede apreciar que esta acción afirmativa permitió corregir aspectos vinculados con el acceso a la carrera, pudo romper la tendencia que excluía de sus aulas a estudiantes de escasos recursos del sector municipal (Castro et al., 2012). Sobre la permanencia destaca el hecho de que son razones vocacionales las que llevan a sujetos que han ingresado vía PSU a desistir de sus estudios. Por otro lado, la mayoría de quienes ingresaron vía cupo de equidad se mantienen en el programa, y si bien se debe profundizar en las razones de salud que aparecen en sus postergaciones, no surgen eliminaciones por temas académicos, contradiciendo lo que muchos detractores de este tipo de medidas señalan al argumentar en contra de la implementación de acciones afirmativas en la enseñanza superior (Castro et al., 2012). En relación al logro académico, al comparar las calificaciones de los estudiantes de los tres 
primeros quintiles que entraron a través del sistema de admisión regular con los de los estudiantes que ingresaron a través del cupo de equidad, muestra que no hay grandes diferencias entre los grupos en ninguna de las asignaturas obligatorias en el primer semestre de 2010. Asimismo, se observa que los/as estudiantes que ingresan vía equidad se demoran dos años en equiparar sus calificaciones a las de sus pares, lo que ha sido ya descrito en la experiencia del Propedéutico de la Universidad de Santiago (Castillo y Cabezas, 2010). Esto pone en evidencia las limitaciones en esta mutua adaptación entre institución y estudiantes, que se expresaría en la reproducción de desigualdades (Neves de Azevedo, 2013), ya sea en los resultados PSU o más tarde en las calificaciones universitarias. Para los/las estudiantes resultan visibles y valorables las estrategias de adaptación de la institución y los mecanismos de apoyo creados para favorecer el aprendizaje y participación de todos los/las estudiantes (Castro et al., 2012). Aspectos por mejorar son la proximidad personal de quienes apoyan, así como también la capacidad del programa para detectar a tiempo las necesidades de los estudiantes, junto con el respecto,la autonomía y participación de estos, por ejemplo, en las estrategias de innovación pedagógica para la diversidad (Castro et al., 2012).

Si bien, se ha logrado mayor equidad en el acceso a la carrera de Psicología, se abre para ésta la necesidad de abordar la equidad en todo el proceso formativo: permanencia, logros y resultados (Espinoza, González y Latorre, 2009).

En base a la experiencia pionera de la carrera de Psicología, a partir del año 2013 se implementa en la Universidad de Chile el Sistema de Ingreso Prioritario de Equidad Educativa de la $\mathrm{UCH}$, consistente en la generación de cupos especiales de ingreso a distintas carreras, reservados para estudiantes provenientes de liceos municipales que pertenezcan a los tres primeros quintiles de ingreso y que cuenten con el puntaje mínimo para postular (600 puntos

Evaluación de la implementación de los cupos de equidad de la Escuela de Obstetricia de la Universidad de Chile.

Andrea Velásquez-Muñoz, Francisco Bustamante-Toncio Maribel Mella-Guzmán, Claudia Cornejo-Aravena DOI : http://dx.doi.org/10.22458/caes.v8i2.1839 
en promedio en la PSU). Entre todos los postulantes se realiza una selección donde se prioriza el Índice de vulnerabilidad de la escuela de origen, luego el quintil de ingreso de la familia del estudiante y en tercer lugar si es perteneciente al $10 \%$ de los mejores promedios de su liceo (Comisión de Equidad Universidad de Chile, 2013).

En el caso de la Facultad de Medicina de la Universidad de Chile, a partir del año 2013 ingresan 48 estudiantes en total, repartidos en las ocho carreras de salud (Sobrero, et al., 2014). A la carrera de Obstetricia y Puericultura, a partir del año 2013 entran 5 estudiantes, al año siguiente 6 estudiantes por año.

Es por ello, que cumplido tres años desde la implementación del Sistema de Ingreso Prioritario con Equidad Educativa en la Facultad de Medicina de la Universidad de Chile, y entendiendo que los y las estudiantes no son meros receptores de política, sino sujetos activos de este proceso, es necesario realizar un estudio descriptivo con el propósito de conocer la experiencia de los y las estudiantes que ingresaron por esta vía a la carrera de Obstetricia de la Universidad de Chile.

El propósito de la presente investigación es poder contribuir a anticipar y priorizar recursos en las áreas deficitarias, y así, facilitar la inserción e integración tanto académica como social de los estudiantes que ingresan por cupo de equidad a la Universidad.

\section{Metodología.}

El diseño metodológico utilizado fue estudio de caso.

Evaluación de la implementación de los cupos de equidad de la Escuela de Obstetricia de la Universidad de Chile.

Andrea Velásquez-Muñoz, Francisco Bustamante-Toncio

Maribel Mella-Guzmán, Claudia Cornejo-Aravena

DOI : http://dx.doi.org/10.22458/caes.v8i2.1839 


\section{Participantes.}

Durante el año 2013 ingresaron cinco estudiantes por cupo de equidad y a partir del año 2014 en adelante ingresan seis estudiantes a la carrera de Obstetricia. Por ende, el universo de la investigación estuvo compuesto por 23 estudiantes que ingresaron por cupos de equidad a la carrera de Obstetricia de la Universidad de Chile y que se encontraban matriculados para el año 2016. En total, 13 estudiantes se encontraban vigentes a fines del año 2016.

Además de lo anterior, con el fin de tener la visión del fenómeno casi en su totalidad fue necesario tener la perspectiva del docente, por lo que se procedió a realizar una entrevista semiestructurada a 3 docentes encargados de los cursos de las ciencias básicas de la Facultad de Medicina.

\section{Instrumentos.}

Dadas las ventajas que ofrece el instrumento del cuestionario, se recurre a él como instrumento principal. Para elaborar la primera redacción se siguieron los pasos habituales, tales como definir objetivos, mapa conceptual de variables y selección de preguntas. Finalmente, se presentaron 9 preguntas cerradas con una escala Likert para sus respuestas. Por otra parte, se decidió presentar 10 preguntas abiertas. Los campos que formaron el cuestionario fueron: características personales y sociodemográficas de la muestra, datos académicos, cursos aprobados y reprobados, relaciones interpersonales con los pares, docentes, percepción de los talleres de nivelación o tutorías, dificultades y/o facilitadores encontrados en la Facultad.

Evaluación de la implementación de los cupos de equidad de la Escuela de Obstetricia de la Universidad de Chile. 
Además del cuestionario, se utilizó una entrevista semiestructurada de 3 preguntas, la que se enfocó en conocer la percepción de los docentes sobre las fortalezas, sugerencias y aspectos a mejorar de los estudiantes que ingresaron por esta vía a la Universidad

\section{Procedimiento.}

La aplicación del cuestionario se realizó en una de las oficinas de la Escuela, en diciembre del año 2015 y en diciembre del año 2016, a todos los estudiantes que se encontraban vigentes en la Universidad. Para los estudiantes que habían desertado y/o renunciado a la carrera se le solicitó a la coordinadora del curso, las causas de abandono y el número de reprobados.

Antes de la realización del estudio, se les informó a todos los estudiantes del objetivo de la investigación y se les solicitó de manera anticipada que firmaran el consentimiento, explicándosele que era voluntario y anónimo y que se podían retirar del estudio cuando quisieran.

El cuestionario fue revisado y analizado por tres investigadores, quienes utilizaron el Excel para procesar los resultados.

Posteriormente, se procedió a entrevistar a los tres docentes en la Facultad, previo consentimiento. La entrevista duró en promedio treinta minutos.

Evaluación de la implementación de los cupos de equidad de la Escuela de Obstetricia de la Universidad de Chile. 


\section{Resultados.}

Durante el año 2013 ingresaron por el Sistema de Ingreso Prioritario con Equidad Educativa (SIPEE) a la carrera de Obstetricia cinco estudiantes. Desde el año 2014 en adelante, el cupo se amplió a seis estudiantes.

A fines del año 2016, solo se encontraban cursando la carrera trece estudiantes (56\%) del total de veintitrés cupos, de los cuales uno es de sexo masculino. El restante abandonó la carrera, por motivos vocacionales, excepto una estudiante que fue eliminada de la carrera por temas académicos.

En relación a la ciudad de procedencia, de los nueve estudiantes, dos son de región (25\%) y los restantes de Santiago.

En relación a la situación familiar, todos viven con familiares, de los cuales tres están acompañados de su familia extendida (37,5\%).

Respecto al nivel de estudios del padre, el $62,5 \%$ de los encuestados contestan que es media completa. De los restantes, uno contestó que su padre tiene nivel técnico completo $(12,5 \%)$ y dos con escolaridad básica (25\%). En relación a la madre, el 87,5\% contestó que tenían escolaridad media completa y el restante básica completa (12,5\%).

Frente a la pregunta sobre la actividad laboral que realizan sus padres, el $37,5 \%$ de los estudiantes expresa que ambos trabajan. Del total de los encuestados, el $50 \%$ menciona que el padre es el sostenedor del hogar.

Evaluación de la implementación de los cupos de equidad de la Escuela de Obstetricia de la Universidad de Chile. 
Del total de los encuestados, el 100\% cursó su educación media en un establecimiento municipal. Con relación a su desempeño durante la enseñanza media, los promedios de notas oscilan entre un 5,8 y un 6,5 . El $37,5 \%$ menciona haber obtenido algún lugar por sus notas.

En cuanto a los conocimientos entregados en física y química durante la enseñanza media, el $87,5 \%$ responde que tuvo clases regulares de física. El 100\% menciona que recibió los contenidos de química. Sin embargo, entre los eventos que perjudicaron su aprendizaje están los paros estudiantiles, los cuales comprimieron los contenidos entregados por los profesores en la enseñanza media, privilegiando la cantidad por la calidad.

Respecto a la PSU, los puntajes van desde 600 hasta 690 puntos aproximadamente. Al postular a una carrera, el $75 \%$ eligió la carrera de Obstetricia como primera opción. El restante postuló a otras carreras de la salud.

En relación a su rendimiento académico en la Universidad, durante los primeros 4 semestres, el $75 \%$ reprobó al menos un curso de las Ciencias Básicas. A pesar de ello, el 100\% de los estudiantes están satisfechos con la carrera de Obstetricia.

El 25\% de los estudiantes expresa que las relaciones interpersonales con los docentes y con sus pares son insatisfactorios.

Con relación a las unidades de apoyo de la Facultad, el 37,5\% de los encuestados acudió al CAPS (Centro de Atención Psicológica), y el 87,5\% acudió al menos una vez al SEMDA (Servicio Médico y Dental).El 25\% de los estudiantes no realiza actividades extracurriculares. Los restantes dedican su tiempo libre a actividades religiosas y artísticas.

Evaluación de la implementación de los cupos de equidad de la Escuela de Obstetricia de la Universidad de Chile.

Andrea Velásquez-Muñoz, Francisco Bustamante-Toncio Maribel Mella-Guzmán, Claudia Cornejo-Aravena DOI : http://dx.doi.org/10.22458/caes.v8i2.1839 
Respecto a la experiencia de ingreso por SIPEE, el 87,5\% recomienda la vía de admisión, sin embargo, cinco estudiantes consideran que la Universidad es demasiado exigente en relación a contenidos teóricos, ya que mencionan que varios contenidos de genética, química y matemática no fueron enseñados durante la enseñanza media. Lo anterior, se evidencia en la alta reprobación que tienen en aquellos cursos, en comparación al resto de los estudiantes. Los estudiantes expresan que una de las principales fortalezas de los cupos de equidad es favorecer el acceso a la educación superior, a propósito de no tener los medios económicos suficientes para complementar sus estudios con un preuniversitario. Además, se menciona que el ingreso por esta vía hace sentir al estudiante capaz de lograr su objetivo "ingresar a la universidad", para muchos es visto como inalcanzable.

Entre las debilidades, se destaca principalmente el déficit de conocimientos teóricos en las Ciencias Básicas. Además, expresan la falta de seguimiento u orientación que garantice la permanencia en la Universidad.

El $100 \%$ de los estudiantes responde que los facilitadores esenciales para su permanencia en la Universidad han sido las redes de apoyo (familia y compañeros de curso) que los motivan y les brindan contención emocional. Por otro lado, destacan la vocación como un elemento esencial para lograr sus objetivos propuestos.

Entre los obstaculizadores para su desempeño académico, los estudiantes comentan que se debe principalmente a la falta de hábitos de estudio, la desorganización en sus tiempos, conocimientos insuficientes sobre las ciencias básicas que adquirieron durante la educación media y la falta de compañerismo entre sus pares. Además, consideran que la excesiva carga académica les ha traído como consecuencia desconcentración, agotamiento físico y 
emocional. Cabe señalar que seis estudiantes refieren que la inseguridad es un elemento obstaculizador, ya que frente a las clases y evaluaciones se sienten con muchas dudas, además de sentirse inferiores en relación a contenidos que se supone deberían haber visto en la enseñanza media. Situación que les causa inseguridad, especialmente, cuando se comparan con sus pares provenientes de colegios de élite, quienes manifiestan mayor dominio de contenidos teóricos.

Para la Escuela de Obstetricia, siempre ha sido fundamental propender a la inclusión y la diversidad, velando en lo posible por un trato igualitario, por lo que muchas veces, no era de conocimiento de los profesores encargados de las Ciencias básicas, las vías de ingreso de cada estudiante. A menos, que la situación lo justificara.

Solo en una oportunidad, un docente planteó una estrategia que quería implementar solo con los estudiantes que ingresaban por SIPEE. Lo cual implicaba que este docente una vez a la semana se reunía solo con los seis estudiantes que habían ingresado ese año e impartía la clase a éste grupo. De acuerdo, a los estudiantes y docente no fue una experiencia satisfactoria, ya que solo permitió corroborar una vez más, las falencias (carencias) de contenidos y las estrategias metodológicas deficientes (estudios memorísticos) que traían los estudiantes, aumentando la angustia y frustración en ellos.

De acuerdo a los docentes entrevistados, las principales fortalezas de los estudiantes que ingresan por cupos de equidad (SIPEE) a la Facultad, fueron la motivación, el entusiasmo y la tolerancia a la frustración. Los aspectos a mejorar,principalmente,se relacionaban con el déficit en los contenidos de las Ciencias básicas (matemática, biología, física y química).

Evaluación de la implementación de los cupos de equidad de la Escuela de Obstetricia de la Universidad de Chile.

Andrea Velásquez-Muñoz, Francisco Bustamante-Toncio Maribel Mella-Guzmán, Claudia Cornejo-Aravena DOI : http://dx.doi.org/10.22458/caes.v8i2.1839 
En base a lo anterior, las sugerencias de los docentes estaban relacionadas con mejorar los programas de nivelación, programas de apoyo y tutorías de la Facultad. Mencionaban que todos los programas de apoyo académico que ayudara a nivelar al estudiante que ingresa por vía SIPEE, debería iniciar en enero y no de manera paralela en marzo o abril, produciendo solo una sobrecarga en los estudiantes, quienes debían quedarse hasta las 19 o 20 horas, que era cuando se terminaban los talleres de nivelación, en vez de las 17 horas.

Todos los docentes concordaban en que no es suficiente el apoyo que realiza la Universidad con los estudiantes que ingresan por SIPEE, ya que los vacíos que traen no se pueden reparar en un par de meses. Necesitan un apoyo académico constante, sistemático que les permita primero integrar los contenidos mínimos obligatorios de la enseñanza media para luego poder asimilar y comprender la lógica de los contenidos en la Universidad.

\section{Discusión.}

En base a los resultados entregados, se puede ver que los trece estudiantes que cursan actualmente la carrera provienen de un nivel socioeconómico vulnerable, provenientes de colegios municipales, donde en la mayoría de las familias no hay estudios superiores y sólo el padre se desempeña en labores remuneradas. Es importante referir que estos estudiantes presentaron un desempeño académico destacado durante la enseñanza media, de manera quela mayoría de ellos fueron reconocidos por su rendimiento, y obtuvieron lugares destacados dentro del curso.

Los estudiantes que permanecen actualmente en la carrera obtuvieron en la Prueba de selección universitaria (PSU) 600 a 660 puntos. Continúan satisfechos con la elección, ya que

Evaluación de la implementación de los cupos de equidad de la Escuela de Obstetricia de la Universidad de Chile.

Andrea Velásquez-Muñoz, Francisco Bustamante-Toncio

Maribel Mella-Guzmán, Claudia Cornejo-Aravena

DOI : http://dx.doi.org/10.22458/caes.v8i2.1839 
refieren ingresar por vocación, sin embargo, llama la atención el alto porcentaje de estudiantes que desertan por vocación, lo que concuerda con las deserciones presentadas en la evaluación de cupos de equidad realizada en la Escuela de psicología de la misma Universidad. Quizás una de las explicaciones se debe a que en la mayoría de los colegios o liceos municipales, no existe una orientadora. Además, se puede desprender de los comentarios de los estudiantes, que su anhelo o sueño principal era entrar a la Universidad de Chile, por su prestigio y reconocimiento a nivel Nacional e Internacional. Por ende, entrar a la carrera de Obstetricia fue el medio para obtener su deseo.

Durante su primer año manifestaron no conocer mucho de la carrera, ni a que se dedicaban las matronas o matrones, pero a medida que pasaron los años se fueron encantando con la profesión y actualmente es uno de los motores que los impulsa para seguir luchando.

De los estudiantes que permanecen (trece), once han reprobado un curso, lo que ha significado que la mayoría presente atraso de por lo menos un año; en el caso de la Escuela de psicología ha habido postergaciones, pero asociadas más bien a temas de salud. En el caso del presente estudio, las reprobaciones de los estudiantes están asociadas al déficit de los conocimientos teóricos en matemáticas, física y química y a la falta de hábitos de estudio, debido a que reconocen dificultades durante su primer y segundo año en la Universidad, principalmente, relacionadas con las falencias (carencias) teóricas que traían de la enseñanza media, asociado a sentimientos de frustración e impotencia, por no obtener las calificaciones y el reconocimiento que estaban acostumbrados a tener durante su educación media. Por el momento, solo una estudiante fue eliminada por temas académicos, lo que no coincide con lo ocurrido en la Escuela de Psicología.

Evaluación de la implementación de los cupos de equidad de la Escuela de Obstetricia de la Universidad de Chile.

Andrea Velásquez-Muñoz, Francisco Bustamante-Toncio Maribel Mella-Guzmán, Claudia Cornejo-Aravena DOI : http://dx.doi.org/10.22458/caes.v8i2.1839 
A pesar de las exigencias y sobrecarga académica, solo un bajo porcentaje consultó al centro de atención psicológica de la Facultad.

Los estudiantes que permanecen en la carrera, reconocen como principal facilitador de su desempeño la vocación, para ellos estudiar lo que querían antes era un sueño, hoy es una realidad, además refieren que las redes de apoyo (familia y compañeros) y el deseo de superación en algunos, permite que persistan en su meta por ser matrones/as.

Hay que destacar que a diferencia de lo percibido por los estudiantes de Psicología, para los que ingresan por cupo de equidad a la carrera de Obstetricia y para los docentes, son poco visibles las estrategias de adaptación creadas por la Facultad e insuficientes los programas de nivelación implementados por la Universidad. Ellos opinan que los talleres de nivelación inician muy tarde, presentan un nivel superior y que las estrategias metodológicas utilizadas, no permiten la enseñanza-aprendizaje, más bien sobrecargan, desmotivan y visibilizan su déficit de conocimientos sobre contenidos que se supone deberían estar adquiridos cuando ingresan a la Universidad.

\section{Conclusiones.}

Se aprecia que durante primer año, bajo el supuesto de que todo el alumnado presentaría similar capacidad, la carrera no lograría ofrecer todos los mecanismos de enseñanza, también llamados "de apoyo" desde una perspectiva socioconstructivista (Coll y Solé, 2002), que son necesarios para el máximo aprendizaje, lo que se reflejaría en las calificaciones del estudiantado. Se podría hipotetizar, un déficit de la institución al momento de educar, en vez de equiparar el desempeño académico, solo reproducen las desigualdades de acuerdo al nivel socioeconómico y tipo de institución. Esto no es percibido en el estudio realizado en la carrera de Psicología (Castro et al, 2012) donde para los estudiantes resultan visibles y valorables las

Evaluación de la implementación de los cupos de equidad de la Escuela de Obstetricia de la Universidad de Chile.

Andrea Velásquez-Muñoz, Francisco Bustamante-Toncio Maribel Mella-Guzmán, Claudia Cornejo-Aravena DOI : http://dx.doi.org/10.22458/caes.v8i2.1839 
estrategias de adaptación de la institución y los mecanismos de apoyo creados para favorecer la enseñanza.

Los estudiantes que ingresan vía cupo de equidad a la carrera de Obstetricia y Puericultura están muy agradecidos y se sienten bendecidos. Lo anterior, es percibido por los docentes, sin embargo, al pasar los años se van dando cuenta que solo existió preocupación para que ingresaran, pues la mayoría se encuentra atrasado un año, lo que implica que el quinto año de la carrera se lo deben costear ellos. A medida que avanzan los años, perciben que solo el esfuerzo y el amor a la carrera, siempre superior a sus pares, les permite permanecer en esta. Perciben ciertas ayudas de la Facultad de Medicina, en relación a tutorías y nivelaciones, sin embargo, son insuficientes y no están diseñadas para ellos, sino más bien para estudiantes que tienen un nivel superior, por lo que desertan de asistir a los talleres, pues sienten que solo los desmotiva y frustra.

Se puede ver, que los estudiantes que ingresan por cupo de equidad a la Escuela de Obstetricia son percibidos por los docentes con competencias actitudinales óptimas (motivados, tolerantes y con vocación), valores y destrezas que les permiten sobrellevar las exigencias y sobrecarga académica. De acuerdo a los propios estudiantes, los principales facilitadores han sido el deseo de superación y el apoyo de sus padres.

En síntesis, se puede evidenciar que, en la Universidad de Chile, se está trabajando para que exista equidad en el acceso a la educación superior como menciona Espinoza et. al. (2009). Sin embargo, no es suficiente. Es necesario repensar los programas de nivelación y tutorías implementados en la Facultad, de manera que sean percibidos como de utilidad y no como sobrecarga académica, convirtiéndose en una fuente generadora de estrés. Los estudiantes 
que ingresan por SIPEE necesitan un apoyo constante y sistemático que los ayude no solo a asimilar conocimientos, sino también a incorporar estrategias metodológicas que faciliten su proceso de enseñanza-aprendizaje, y así, contribuir a generar herramientas que faciliten la permanencia y el egreso.

La educación superior debe asegurar no solo el derecho a la educación para todos, sino también debe asegurar la equidad y calidad de la educación (Unesco, 1998; Espinoza et al., 2009).

\section{Referencias}

Bellei, C.\& Huidobro, J. (2003). Desigualdad educativa en Chile. Santiago, Chile: Departamento de Educación, Universidad Alberto Hurtado.

Castillo, J.\& Cabezas, G. (2010). Caracterización de jóvenes de primera generación en educación superior. Nuevas trayectorias hacia la equidad educativa. Revista Calidad en la educación (32), 54-74. Recuperado el 6 de abril de 2016 desde http://www.cned.cl/public/secciones/SeccionRevistaCalidad/doc/67/cse ar $\underline{\text { ticulo900.pdf }}$

Castro, P.,Antivilo, A., Aranda, C., Castro, C., Lizama, C., Williams, J.\& De Torres, H. (2012). El efecto de la implementación del "cupo de equidad" en la carrera de Psicología de la Universidad de Chile. Revista de Inclusión Social y Equidad en la Educación Superior (10), $167-168$

Cepal (2010). Panorama social de América Latina. Recuperado de http://www.cepal.org/publicaciones/xml/9/41799/PSE-panoramasocial2010.pdf.

Coll, C. y Solé, I. (2002). "Enseñar y aprender en el aula". Capítulo 14 en: Comp. Coll, C., Palacios, J. y Marchesi, A. Desarrollo psicológico y educación, tomo II, colección Psicología y Educación. Alianza Editorial.

Evaluación de la implementación de los cupos de equidad de la Escuela de Obstetricia de la Universidad de Chile.

Andrea Velásquez-Muñoz, Francisco Bustamante-Toncio

Maribel Mella-Guzmán, Claudia Cornejo-Aravena

DOI : http://dx.doi.org/10.22458/caes.v8i2.1839

Artículo protegido por licencia CreativeCommons 
Comisión de Equidad Universidad de Chile (2013). Memoria 2010-2013 Equidad y Calidad: el compromiso de la Universidad de Chile con el país. Santiago de Chile: Universidad de Chile.

Comisión Equidad Universidad de Chile (2014). Política de equidad e inclusión en la Universidad de Chile. (Informe al Consejo Universitario). Santiago de Chile: Universidad de Chile.

Contreras, M., Corbalán, F.\& Redondo, J. (2007). Cuando la Suerte está Echada: Estudio Cuantitativo de los Factores Asociados al Rendimiento en la PSU. REICE - Revista Electrónica Iberoamericana sobre Calidad, Eficacia y Cambio en Educación, vol. 5(5e), 259-263. Recuperado el 15 de abril de 2016 desde http://www.redalyc.org/pdf/551/55121025031.pdf

Espinoza, O., González, L. \& Latorre, C. (2009). Un modelo de equidad para la educación superior: Análisis de su aplicación al caso chileno. Revista de la educación superior,38(150), 97-111. Recuperado el 31 de mayo de 2016 desde http://www.scielo.org.mx/scielo.php?script=sci arttext\&pid=S0185$27602009000200006 \& \operatorname{lng}=e s \& t \operatorname{lng}=\mathrm{pt}$.

Neves de Azevedo, M. (2013) Igualdade e Equidade: qual é a medida da justiça social? Avaliação, 18(1), 129-150. Recuperado el 16 de mayo de 2016 desde http://submission.scielo.br/index.php/aval/article/view/113712

OCDE Organización para la Cooperación y el Desarrollo Económico. (2004). Chile. Revisión de políticas nacionales de educación. París: OECD Publishing.

OCDE Organización para la Cooperación y el Desarrollo Económico. (2009). La educación superior en Chile. Recuperado de http://www.oecd-ilibrary.org/laeducacion-superior-enchile_5ksntst3t7hd.pdf

Pérez, V. (2009). Nuevo trato con el Estado. Hacia una política para las universidades estatales. Documento de trabajo.

Pérez, V. (2011). Discurso en inauguración de año académico 2011. Recuperado de http://www.uchile.cl/.

Evaluación de la implementación de los cupos de equidad de la Escuela de Obstetricia de la Universidad de Chile.

Andrea Velásquez-Muñoz, Francisco Bustamante-Toncio

Maribel Mella-Guzmán, Claudia Cornejo-Aravena

DOI : http://dx.doi.org/10.22458/caes.v8i2.1839

(c)

Artículo protegido por licencia CreativeCommons 
Sobrero, V., Lara, R., Méndez, P.\& Suazo, B. (2014). Equidad y diversidad en universidades selectivas: la experiencia de estudiantes con ingresos especiales en las carreras de la salud. Pensamiento Educativo. Revista De Investigación Educacional Latinoamericana, 51(2), 152-164. Recuperado el 31 de mayo de 2016 de http://pensamientoeducativo.uc.cl/index.php/pel/article/view/669

Solimano, A. \&Torche, A. (2008). La distribución del ingreso en Chile 1987-2006: análisis y consideraciones de política (Documento de trabajo $\mathrm{n}^{\circ} 480$, Banco Central de Chile). Recuperado de http://www.bcentral.cl/esp/estpub/estudios/dtbc

UNESCO (1998). Tendencias de la Educación Superior en América Latina y el Caribe. Caracas: IESALC-UNESCO.

Valenzuela, J., Bellei, C. \& De Los Ríos, D. (2009). Evolución de la segregación socioeconómica de los estudiantes chilenos y su relación con el financiamiento compartido. En Mineduc (Ed.), Evidencias para Políticas Públicas en Educación (231284). Santiago, Chile: FONIDE, Ministerio de Educación.

Williams, J. (2013). Discursos de estudiantes sobre su propia situación social y educativa en programas de equidad en universidades selectivas. (Tesis de Magíster). Facultad de Ciencias Sociales. Universidad de Chile.

Evaluación de la implementación de los cupos de equidad de la Escuela de Obstetricia de la Universidad de Chile. 